\title{
NUMERICAL STUDY OF THE FLUID DYNAMICS OF A MICRO PILLAR IMMERSED IN A LAMINAR BOUNDARY LAYER
}

\author{
M. I. Yaras ${ }^{1}$, C. Metin ${ }^{1}$ \\ ${ }^{1}$ Dept. of Mechanical and Aerospace Engineering, Carleton University, Ottawa, Canada
}

\begin{abstract}
The study investigates the flow development in the vicinity and wake of a micro pillar mounted on a surface immersed in the inner region of a laminar boundary layer. The investigation is based on the numerical solution of the Navier Stokes equations for incompressible conditions. It is demonstrated that the significant wall-normal gradient of streamwise velocity of the cross flow spanning the height of the micro pillar results in suppression of time-periodic shedding of vorticity from the side surfaces of the micro pillar, resulting in a steady wake flow. A pair of streamwise-oriented tip vortices and a corresponding pair of base vortices are observed to dominate the wake development. The sources of vorticity for these vortices and their relation to the vorticity in the recirculation zone immediately downstream of the micro pillar are identified. This improved understanding of flow physics is intended to inform future studies of passive control of boundary layers.
\end{abstract} layers

Keywords- cylinder in crosslflow; wake vorticity; boundary

\section{INTRODUCTION}

The coherent vortical structures associated with a surfacemounted finite cylinder in a two-dimensional boundary layer are known to be influenced primarily by: the state (turbulent vs. laminar) of the base-surface boundary layer; the height-todiameter ratio (aspect ratio) of the cylinder; the flow Reynolds number based on the characteristic dimension of the cylinder; and, the thickness of the base-surface boundary layer relative to the cylinder height. These vortical structures can be broadly characterized by three systems: (I) the base vortices and the horseshoe vortex near the cylinder/base-surface junction; (II) vorticity shedding along the height of the cylinder upon separation of the side-surface boundary layer; and, (III) the tip vortex system that is located at the free end of the cylinder. The vorticity source for the horseshoe vortex is the spanwiseoriented vorticity of the undisturbed boundary layer approaching the cylinder along the base surface. This vorticity field concentrates on the windward side of the cylinder under the stagnating influence of the pressure field of the cylinder, with the vorticity vector of the affected fluid particles tilting to develop a streamwise component as they are displaced laterally and vertically from their undisturbed trajectory. The resultant coherent vortical structure at the base of the cylinder is typically referred to as the horseshoe vortex owing to its commonly observed shape [1-4]. On the leeward side of the cylinder, the counter-rotating streamwise-oriented legs of the horseshoe vortex may be accompanied by a second pair of counter-rotating vortices near the base of the cylinder, commonly referred to as the junction or base vortices $[1,2,5]$. The sense of rotation of the base vortices is such that they induce upwash in the wake of the cylinder, which is opposite of the induction effect of the streamwise legs of the horseshoe vortex. The formation of the base vortices has been noted to depend on the aspect ratio of the cylinder and the thickness of the base-surface boundary layer relative to the height of the cylinder. Cylinder aspect ratios larger than a critical value, suggested to be in the 3 to 5 range by Sumner et al. [5], and a relatively thick base-surface boundary layer have been noted to promote the existence of the base vortices [5-7]. The relative strength and characteristics of time-periodic vorticity shedding from the side surfaces of the cylinder depend on the aspect ratio of the cylinder. Below a cylinder aspect ratio of about 2.0 such transient shedding may be suppressed [8], and in the range of 2.0 to 6.0 the periodic shedding is intermittent [9]. At an aspect ratio of 2.5, negligible base surface boundary layer thickness, and a flow Reynolds number based on cylinder diameter of 43,000, Fröhlich and Rodi [10] observed alternating shedding along the lower portion of the cylinder side surface only. Above a critical value of cylinder aspect ratio, which has been observed to depend on the flow Reynolds number and the thickness of the base-surface boundary layer relative to the cylinder height, the boundary layer separating from the side surface of the cylinder rolls asymmetrically or symmetrically from the port and starboard sides into vortical structures in a time-periodic manner that results in the wellknown Karman vortex street $[9,11]$. For low aspect ratios, this vortex shedding appears to be suppressed by the downwash and upwash motions prevailing on the leeward side of the cylinder induced by the vortical structures that exist near the free end and base of the cylinder $[1,5,9,11,12]$. The vortex system near

This study was funded by the Kenneth Molson Foundation. 
the free end of the cylinder is somewhat more complex owing to the interaction of the vorticity associated with the top and side surfaces of the cylinder [13]. The geometric discontinuity at the intersection of the top and side surfaces prompts flow separation along the windward edge of the top surface. For cylinders with low aspect ratios, reattachment to the top surface follows $[6,7,9,11,14]$. Otherwise, the vorticity associated with this separated flow amalgamates into an arch- or mushroomshaped vortex, with its main portion oriented in the spanwise direction and its legs extending to two focal points on the base surface $[6,7,9,10,15,16]$. The legs of the arch vortex have been observed to also merge with a pair of streamwise oriented, counter-rotating tip vortices that emerge from two foci on the rear of the cylinder near the leeward edge of the cylinder top surface [10]. The tip vortices were also identified as the dominant vortical structures on the free end of the cylinder by Hain et al. [14]. The relative strengths of the tip and base vortex pairs dictate whether only the tip vortices (dipole configuration) or both the tip and base vortices (quadrupole configuration) prevail in the wake of the cylinder [13]. The prevailing scenario has been observed to depend on the aspect ratio of the cylinder as well as flow Reynolds number, with recent evidence on the existence of a topology involving six vortices at certain Reynolds numbers [17]. It has also been suggested that in the far wake, the streamwise vortex pairs identified in the time-averaged vorticity field as being respectively positioned near the base surface and at a distance comparable to the cylinder height may be the streamwise and spanwise connector strands that span between the vortical structures which form through time-periodic shedding of vorticity from the side surface of the cylinder $[15,18]$.

The surface-mounted cylinder configurations that have been studied to date have tended to place the free end of the cylinder either in the upper portion or beyond the edge of the boundary layer. In a two-dimensional boundary layer developing under zero or favourable streamwise pressure gradients, majority of the boundary-layer vorticity is confined to the inner region of the boundary layer. Based on the flow physics reviewed in the foregoing, placing the free end of the cylinder within this high-vorticity region may yield dynamics that deviate from the trends observed for cylinders where the undisturbed-flow vorticity near the free end of the cylinder is either low or zero. Improved understanding of this configuration is important for informing future design optimization studies of micro pillars intended to affect the streamwise development of boundary layers.

\section{COMPUTATIONAL METHOD}

The computational domain and boundary conditions are illustrated in Fig. 1. The domain is sized to allow for the flow field in the vicinity of the micro pillar and its wake to develop without any artificial influence by the imposed boundary conditions. The positioning of the outflow boundary relative to the location of the micro pillar is also driven by the need to have sufficient distance for the wake region to develop downstream of the micro pillar.

The laminar-flow velocity profile at the inflow boundary corresponds to the velocity profile of an equilibrium turbulent boundary layer under zero streamwise pressure gradient, at a Reynolds number based on momentum thickness of 900. Such a velocity profile can be realized in a laminar boundary layer via favourable streamwise pressure gradients of suitable magnitudes upstream of the inflow boundary of the present domain. In wall units, based on the wall shear stress at the axial location of the micro pillar, the micro-pillar diameter has a value of $\mathrm{D}^{+}=18$. For $\mathrm{H} / \mathrm{D}=4.0$, the corresponding normalized micro-pillar height is $\mathrm{H}^{+}=72$. If the base-surface boundary layer were turbulent, $\mathrm{H}^{+}=72$ would place the top surface of the micro pillar in the lower portion of the log-law region. This is to illustrate that the micro-pillar is sized to place its free end in a region of significant boundary-layer vorticity.

The spatial grid is based on a structured-grid layout of hexahedral cells. For a well-controlled distribution of the grid cell size, the domain is subdivided into multiple blocks in the streamwise, spanwise and wall-normal directions as shown in Fig. 2. The smallest grid cells with a size of $0.01 \mathrm{D}$ are located in the vicinity of the micro-pillar. This, together with the mild rate of spatial gradients in the cell sizes as illustrated by the $\mathrm{R}$ parameter in Fig. 2, results in a total grid-cell count of 4.6 million in the domain. This spatial grid yields a very conservative spatial resolution of the flow field. This was confirmed through a grid-sensitivity analysis.

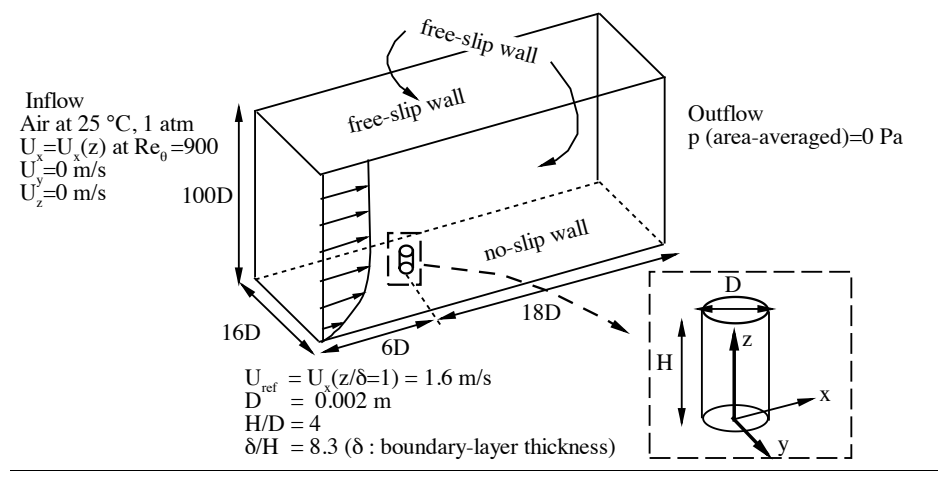

Figure 1 Computational domain and boundary conditions.

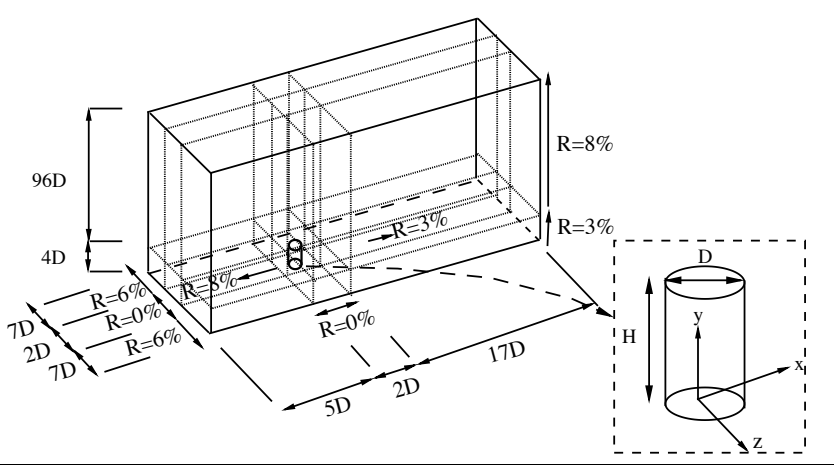

Figure 2 Grid-block topology (R: grid-cell growth rate). 
The time-step size normalized by $\mathrm{U}_{\text {ref }}$ and $\mathrm{D}$ is set to 0.002 . This time increment yields a Courant number value of less than 0.5 for any of the grid cells in the domain, which is sufficient for numerical stability as an under-relaxation parameter and for resolving possible time-periodic shedding of vorticity in the wake of the micro pillar. The chosen time-step size corresponds to approximately $1 / 75$ th of the voricity shedding period in the wake of the micro pillar if the micro pillar wake were to develop in a manner similar to the wake of a two-dimensional cylinder in uniform cross flow.

The commercial software package ANSYS CFX 13.0 is used to solve the continuity and momentum equations governing the fluid flow. The authors' research group has used and validated the solution algorithm of this software extensively in direct numerical simulations of attached and separated shear layers in transitioning and turbulent states [19]. The governing equations are spatially discretized using a finite volume approach. Spatial derivatives are evaluated using a spatial interpolation scheme that is equivalent to second-order centered-differencing for the diffusion term, and a blend of first-order of upwind and second-order centered differencing for the convection term. Second-order Euler backward differencing is used for temporal derivatives. Benchmark simulations performed by the authors' research group have shown a root-mean-square normalized residual value on the order of $10^{-5}$ to be adequate for satisfactory convergence. Convergence of the discretized equations to this residual value is realized through up to eight outer-loop iterations at each time step, which converge the coefficients of the linearized equations and a single inner-loop iteration per outer-loop iteration in which the linearized equations are solved through a W-type algebraic multigrid cycle. The multigrid cycle uses six grid levels, with one and three solution sweeps performed at each grid level during the restriction and prolongation passes of each cycle, respectively.

\section{RESULTS AND DISCUSSION}

The Reynolds number based on the micro-pillar diameter, $\mathrm{D}$, and the undisturbed boundary layer velocity at the streamwise $(\mathrm{x})$ location of the micro pillar at $\mathrm{z}=\mathrm{H}$ distance from the base surface is 125 . Given the wall-normal velocity gradient, this Reynolds-number value would be 68 if it were based on the velocity spatially averaged over the height of the micro pillar rather than the value at $\mathrm{z}=\mathrm{H}$. A $2 \mathrm{D}$ (i.e. infinitely long) cylinder in uniform cross flow at these flow Reynolds number values falls in the 40-150 Reynolds-number range for which the cylinder would experience transient alternating vortex shedding from its starboard and port sides to produce the well-known Karman vortex street. The present simulation results indicate a steady flow field for all the configurations considered. The aspect ratio of the micro pillar may affect this outcome. A height-to-diameter ratio of 2.0 has been suggested in the published literature as the threshold below which periodic shedding in the wake is suppressed. The H/D ratio of the present micro pillar is double of this threshold. It is therefore interesting to observe suppression of the absolute instability mechanism driving the periodic shedding of vortices. The micro pillar in the present study is placed in a cross-flow with a velocity gradient that is significantly higher along the full height of the micro pillar than the cross-flow conditions considered in published literature. It may therefore be argued that this gradient plays a significant role in suppression of periodic shedding from the micro pillar. Analysis of the flow field around the micro pillar and its wake as presented in what follows may help to further explore this potential cause-and-effect relationship.

The flow field in the vicinity and wake of the micro pillar is presented through plots of several parameters in Fig. 3. Part (a) of the figure presents the limiting flow streamlines on the top and side surfaces of the micro pillar, which are in essence skin friction lines. Part (b) presents the skin friction lines on the base surface ( $\mathrm{z}=0$ plane) and the streamlines on the $\mathrm{y}=0$ plane together with the static pressure distributions in these planes illustrated as a flood plot of the static pressure coefficient defined as $\left(\mathrm{P}-\mathrm{P}_{\text {ref }}\right) / 0.5 \rho \mathrm{U}_{\text {ref }}^{2}$, where the reference condition corresponds to the undisturbed freestream condition. The plotted range for this pressure coefficient is based on the pressure range realized within the $\mathrm{x}, \mathrm{y}$ and $\mathrm{z}$ bounds of the plot. Part (c) presents isosurfaces of the second invariant of the velocity gradient tensor (Q), which has been established to be an effective flow parameter to visualize coherent vortical structures. The isosurfaces correspond to a nondimensional Q value of 0.00375 , for which the reference velocity and the micro-pillar diameter are used for normalization. This value was determined to be most effective in visualizing the dominant vortical structures while avoiding the clutter associated with smaller and substantially weaker vortical flow features.

The surface skin-friction-line pattern on the micro pillar suggests the presence of several singular points, which develop at surface locations where the wall shear stress or the surface vorticity becomes identically zero. Conventionally such singular points are identified as either saddle points or nodes, and nodes are further subcategorized as either nodal points or foci of attachment or separation [20]. At a nodal point, all but one of the skin friction lines converging to that point become tangent to a single line passing through the point. Whether the lines are directed towards or away from the point depends on whether the nodal point corresponds to flow separation or attachment, respectively. A focal point differs from a nodal point in that the skin friction lines do not have a common tangent at such a singular point; instead, they converge at that point in a spiral pattern. For example, a vortex connecting with a surface would yield a focal point. At a saddle point, only two skin friction lines intersect, with the skin friction directed towards the point on one of the lines, and away from it on the other. All other skin friction lines in the vicinity avoid this point, with the two intersecting skin friction lines in effect acting as barriers for these remaining lines. With these definitions, identification of nodal, focal and saddle points on the micro-pillar surface shed considerable light on the flow field around the micro pillar. 
a)
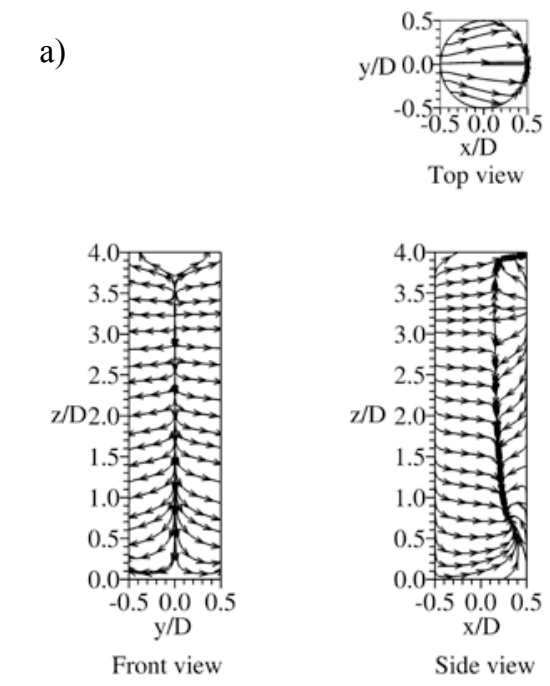

b)

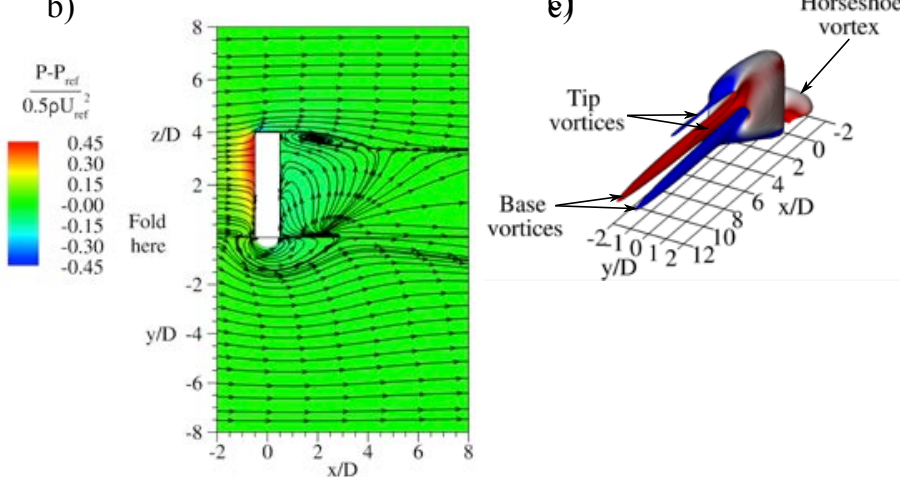

Figure 3 Flow field in the vicinity and wake of the micro pillar: a) micro-pillar surface streamlines; b) pressure field and streamlines in $\mathrm{y}=0 \mathrm{\&} \mathrm{z}=0$ planes; c) $\mathrm{Q}=0.00375$ isosurfaces

In Fig. 3a, the front view of the micro pillar shows a nodal point near the free end of the micro pillar and a saddle point at the base, with both points positioned in the $\mathrm{y}=0$ plane. For a 2D cylinder in uniform cross-flow, the cross-flow stagnates on the cylinder surface in this plane. Because the micro pillar is placed in a cross-flow with a large gradient of streamwise velocity along the height of the micro-pillar, as the micropillar is approached in the symmetry $(\mathrm{y}=0)$ plane the static pressure recovery is larger for the fluid farther from the base surface. The resultant static pressure field immediately upstream of the micro-pillar, as illustrated in Fig. 3c, drives the fluid in the $\mathrm{y}=0$ plane towards the base of the micro pillar along the lower $3 / 4^{\text {th }}$ of the micro-pillar height. This motion of the fluid is terminated at the base surface, resulting in the development of a saddle point. The fluid in the $\mathrm{y}=0$ plane along the upper $1 / 4^{\text {th }}$ of the micro-pillar height is noted to move upwards. This is due to the fact that the deceleration in the $\mathrm{x}$ direction and hence static pressure recovery of the crossflow is limited approximately to the height of the micro-pillar (Fig. 3c). The resultant switch from downward motion to upward motion along the height of the micro pillar at about $3 / 4$ height results in the formation of a nodal point. The role of the nonuniformity of the cross-flow on this flow pattern is further informed by contrasting the surface streamline pattern in the front view of Fig. 3a with that in Fig. 4a, with the latter corresponding to a spatially uniform undisturbed velocity profile. For the simulation corresponding to the results in Fig. 4 , the inflow velocity profile was set to be uniform and the base surface was set to the free-slip condition. The absence of downward motion in the $\mathrm{y}=0$ plane for the uniform cross-flow conditions, which eliminates the nodal point that is observed near the free end of the micro pillar in the non-uniform crossflow case, and leads to the saddle point near the base surface to become a nodal point, is consistent with the flow physics described in the foregoing.

For a 2D cylinder in cross-flow, the boundary layer along the cylinder circumference starts to develop at the stagnation point on the windward side and separates from the cylinder surface farther along the surface for all but very low Reynolds number values corresponding to creeping flow. The azimuthal position of separation is a function of flow Reynolds number. The side view in Fig. 3a indicates the separation to occur increasingly farther along the micro-pillar surface as the base of the micro pillar is approached. This trend is consistent with the reduction in the local flow Reynolds number with proximity to the base surface, dictated by the velocity gradient in the cross flow. Absence of delayed separation towards the base of the micro-pillar for the uniform cross-flow case illustrated in the side view in Fig. 4a supports this argument. In close proximity of the free end of the micro pillar the location of separation is also delayed. This is driven by the reduced streamwise pressure gradient affected by the threedimensionality of the local flow field. Three dimensionality of the flow results in a three-dimensional separation from the micro-pillar surface, with motion along the line of separation. This motion is observed to be upwards along the upper $1 / 4^{\text {th }}$ of the separation line, and downwards along the remaining length. Fluid motion in close proximity of the micro-pillar side surface on both sides of the separation line is towards the separation line as expected for a 3D separation process, and mimics the upward/downward motions observed along the separation line. This results in the formation of a saddle point about $1 / 4^{\text {th }}$ micro-pillar height from the free end. The separation line is terminated at its lower end by another point of singularity. The spiraling pattern of the surface streamlines suggest this to be a focal point, which is a singular point typically associated with the termination of a vortex at a solid boundary. This vortex is identified as the base vortex in Fig. 3c. This vortex and its counterpart originating on the starboard side are observed to be closely aligned with the streamwise direction and dominate the near-wake flow field. The Q isosurface in Fig. 3c is coloured based on the sign of the $\mathrm{x}$-component of the local vorticity vector. Red colour denotes a counter-clockwise sense of rotation when viewed from downstream, and blue denotes the opposite. The source of the vorticity of the base vortices is the boundary layer that 
develops on the micro-pillar side surface. For a 2D cylinder in uniform cross flow, the vorticity lines in the boundary layer are aligned with the axis of the cylinder. Because of the crossflow velocity gradient along the height of the micro pillar, the vorticity lines in the micro pillar boundary layer deviate from

a)
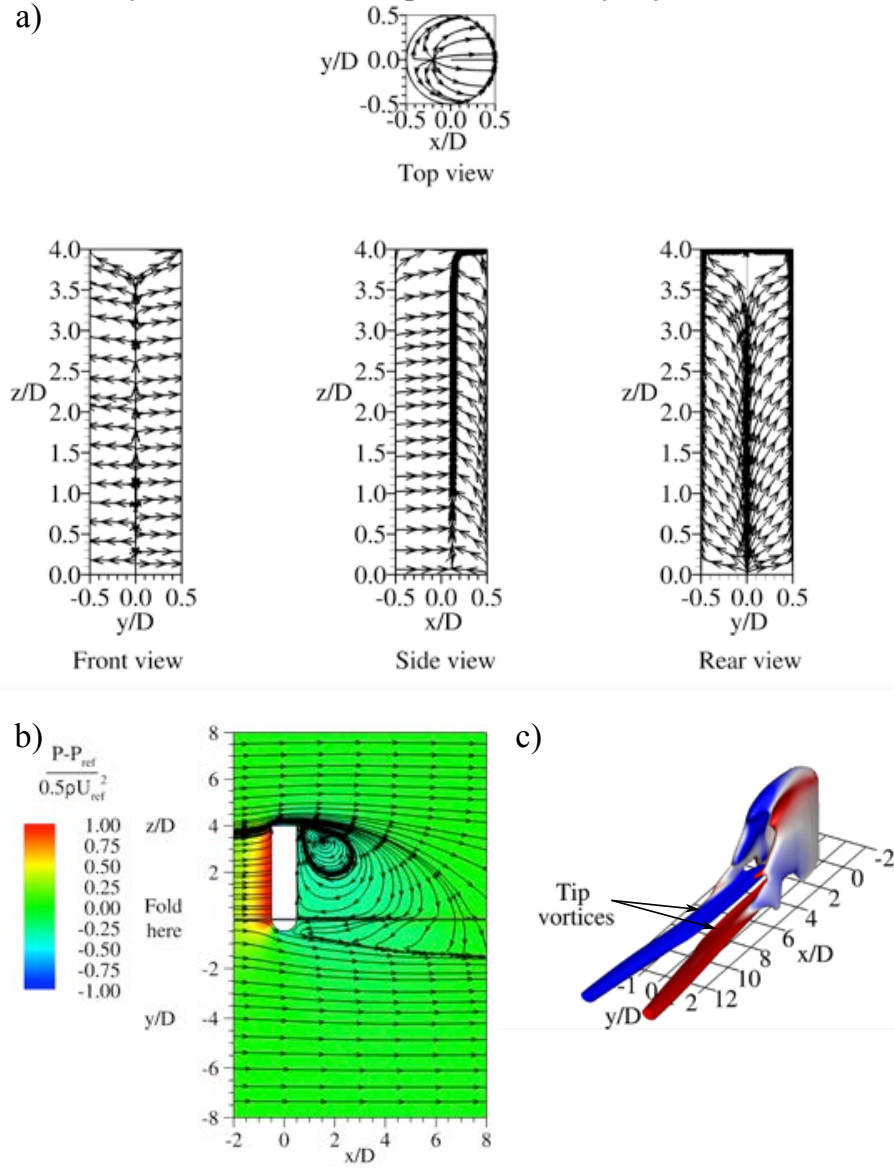

Figure 4 Flow field in the vicinity and wake of the micro pillar in uniform cross-flow: a) micro-pillar surface streamlines; b) pressure field and streamlines in $\mathrm{y}=0$ \& $\mathrm{z}=0$ planes; $\mathrm{c}) \mathrm{Q}=0.00375$ isosurfaces.

the $\mathrm{z}$ direction, approximating a tilting motion towards the downstream direction. This results in a nonzero value for the $\mathrm{x}$ component of this vorticity field, which then develops into a coherent vortex that is identified as the base vortex in Fig. 3c. Based on this interpretation of the flow physics, the base vortices would not develop in absence of the velocity gradient in the cross flow. This is confirmed based on the uniform cross-flow results shown in Fig. 4c. As observed in Fig. 3b, the pair of base vortices induce an upwash on the symmetry plane on the leeward side of the micro pillar. This upward motion is restricted by a recirculation zone located immediately downstream of the free end of the micro pillar.

On the free end of the micro pillar, hereafter referred to as the top surface, the flow separates at the windward edge affected by the first-derivative discontinuity of the surface geometry along the edge. The flow is observed to reattach to the top surface immediately after separation, forming a very small recirculation zone along the edge that is too small to observe in the present figures. The vorticity associated with the boundary layer developing on the top surface is primarily oriented in the spanwise (y) direction as suggested by the surface streamlines in the top view of Fig. 3a. This vorticity sheds off the leeward edge of the top surface. However, this is not the source of the vorticity associated with the recirculation zone visible in the streamline pattern in the $\mathrm{x}-\mathrm{z}$ plane in Fig. $3 \mathrm{~b}$. This was determined through a simulation in which the free-slip condition was imposed on the top surface of the micro pillar. The recirculation zone corresponding to this scenario, shown in Fig. 5b, is very similar to that noted in Fig. $3 \mathrm{~b}$. The vorticity field immediately downstream of the micro pillar close to the free end is instead sourced by the boundary layer developing on the side surface of the micro pillar. The boundary layer fluid on the micro pillar side surface is noted to migrate towards the free end while traversing along the micro-pillar circumference (Fig. $3 \mathrm{a}$ - front and side views), as driven by the prevailing pressure gradients. As per the surface streamlines in Fig 3a - side view, this boundary-layer fluid converges in the flow region immediately downstream of the micro-pillar free end, with the vorticity of this fluid sourcing the recirculation zone observed in the x-z plane in Fig. 3b. The noted upward migration of the boundary-layer fluid results in the vorticity vector to deviate from its alignment with the $\mathrm{z}$ axis, and the resultant $x$ component of vorticity sources the streamwise extensions of the spanwise oriented vorticity in the recirculation zone, which are labeled as tip vortices in Fig. 3c.

Based on the spatial extent of the isosurfaces of Q in Fig. $3 \mathrm{c}$, the tip vortices are observed to be notably weaker than the base vortices upon formation. The velocity field that results from the combined influence of the base vortices, the tip vortices, and the recirculation zone linking the two tip vortices yields a nodal point in the $\mathrm{x}-\mathrm{z}$ plane on the rear side of the micro pillar close to the free end, and a saddle point closer to the base (Fig. 3a, rear view).

In addition to the base and tip vortices, the only other identifiable coherent vortical structure in the flow field is the horseshoe vortex that forms near the base on the windward side of the micro pillar. This vortex is visible in Fig. 3c, and its presence can also be gleaned from the surface streamlines on the base surface and the $x-z$ symmetry plane in Fig. 3b. The horseshoe vortex forms through accumulation of the initiallyspanwise-oriented vorticity lines of the cross-flow boundary layer on the windward side of the micro pillar, which wrap themselves around the micro pillar in the approximate shape of a horseshoe. The surface streamlines on the base surface in Fig. $3 \mathrm{~b}$ show that the $3 \mathrm{D}$ separation line associated with the horseshoe vortex terminates at about two micro-pillar diameters downstream from the micro pillar, which may be interpreted as the streamwise extent of the legs of the horseshoe vortex. Absence of the streamwise legs of the horseshoe vortex with the chosen value of isosurfaces for the Q parameter in Fig. 3c suggests these legs to be notably weaker than the base vortices. Furthermore, based on the sign of the vorticity in each leg of the horseshoe vortex relative to 
the base vortex on the same side of the micro pillar, destructive mutual interaction of these vortices is expected.

a)
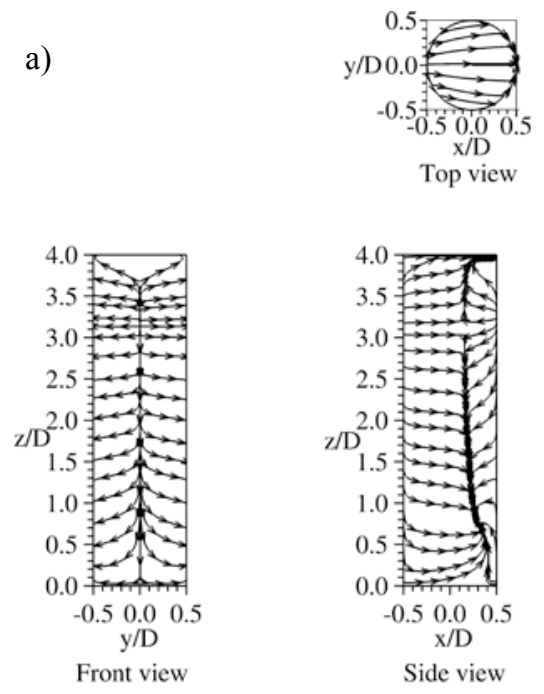

b)

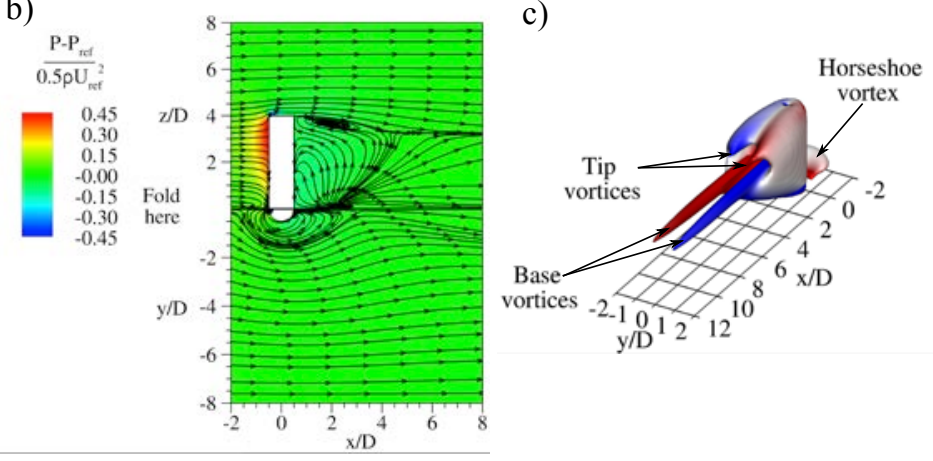

Figure 5 Flow field in the vicinity and wake of the micro pillar with no vorticity production/destruction on the top surface of micro-pillar: a) micropillar surface streamlines; b) pressure field and streamlines in $y=0$ \& $\mathrm{z}=0$ planes; c) $\mathrm{Q}=0.00375$ isosurfaces.

\section{CONCLUSIONS}

Numerical simulations are performed of the laminar flow around a micro-pillar mounted deep inside a zero-streamwisepresure-gradient boundary layer on a flat surface. The height of the micro pillar corresponds to the portion of the boundary layer where the vorticity magnitude is the highest. The development of the flow over the side and top surfaces of the micro pillar is observed to be significantly affected by this vorticity. Time-periodic shedding of vorticity from the side surface of the micro-pillar, that has been observed in published literature for similar flow Reynolds numbers and geometric configurations, is noted to be suppressed by the significant velocity gradient in the cross-flow yielding a steady micropillar wake. The top surface of the micro pillar is shown not to contribute significantly to the vorticity field in the wake. The wake is observed to be dominated by a pair of streamwise tip vortices, a pair of streamwise base vortices, and a recirculation zone positioned in the immediate vicinity of the micro pillar. It is demonstrated that the vorticity generated on the side surface of the micro pillar is the source of vorticity for all of these vortical structures in the micro-pillar wake.

\section{REFERENCES}

[1] G.D. Donnert, M. Kappler and W. Rodi, "Measurement of tracer concentration in the flow around finite-height cylinders," J. of Turbulence, (8), N33, 2007.

[2] A. Sau, R.R. Hwang, T.W. Sheu and W.C. Yang, "Interaction of trailing vortices in the wake of a wall-mounted rectangular cylinder," Physical Review E, 68(5), 056303, 2003.

[3] C. Lin, P.H. Chiu, S.J. Shieh, "Characteristics of horseshoe vortex system near a vertical plate-base plate juncture," Experimental Thermal and Fluid Science, 27(1), pp. 25-46, 2002.

[4] C.J. Baker, "The laminar horseshoe vortex," J. of Fluid Mechanics, 95(2), pp. 347-367, 1979.

[5] D. Sumner, J.L. Heseltine and O.J.P. Dansereau, "Wake structure of a finite circular cylinder of small aspect ratio," Experiments in Fluids, 37(5), pp. 720-730, 2004.

[6] R.J. Pattenden, S.R. Turnock and X. Zhang, "Measurements of the flow over a low-aspect-ratio cylinder mounted on a ground plane," Experiments in Fluids, 39(1), pp. 10-21, 2005.

[7] R.J. Pattenden, N.W. Bressloff, S.R. Turnock and X. Zhang, "Unsteady simulations of the flow around a short surface-mounted cylinder," Int. J. for Numerical Methods in Fluids, 53(6), pp. 895914, 2007.

[8] T. Okamoto and M. Yagita, "The experimental investigation on the flow past a circular cylinder of finite length placed normal to the plane surface in a uniform stream," Bull. JSME 16 (95), pp. 805814, 1973.

[9] T. Kawamura, M. Hiwada, T. Hibino, I. Mabuchi and M. Kumada, "Flow around a finite circular cylinder on a flat plate: cylinder height greater than turbulent boundary layer thickness," Bulletin of JSME, 27(232), pp. 2142-2151, 1984.

[10] J. Fröhlich and W. Rodi, "LES of the flow around a circular cylinder of finite height," Int. J. of Heat and Fluid Flow, 25(3), pp. 537-548, 2004.

[11] D. Sumner, "Flow above the free end of a surface-mounted finiteheight circular cylinder: a review," J. of Fluids and Structures, 43, pp. 41-63, 2013. [14] R. Hain, C.J. Kähler and D. Michaelis, "Tomographic and time resolved PIV measurements on a finite cylinder mounted on a flat plate," Experiments in Fluids, 45(4), pp. 715-724, 2008.

[12] H. Wang, Y. Zhou, C. Chan and T. Zhou, "Momentum and heat transport in a finite-length cylinder wake," Experiments in Fluids, 46(6), pp. 1173-1185, 2009.

[13] Z. Hosseini, J.A. Bourgeois and R.J. Martinuzzi, "Large-scale structures in dipole and quadrupole wakes of a wall-mounted finite rectangular cylinder," Experiments in Fluids, 54(9), pp. 1595$1610,2013$.

[14] R. Hain, C.J. Kähler and D. Michaelis, "Tomographic and time resolved PIV measurements on a finite cylinder mounted on a flat plate," Experiments in Fluids, 45(4), pp. 715-724, 2008.

[15] S. Krajnović, "Flow around a tall finite cylinder explored by large eddy simulation," J. of Fluid Mechanics, 676, pp. 294-317, 2011.

[16] G. Palau-Salvador, T. Stoesser, T., J. Fröhlich, M. Kappler and W. Rodi, "Large eddy simulations and experiments of flow around finite-height cylinders," Flow, Turb. and Comb., 84(2), 239, 2010.

[17] D. Zhang, L. Cheng, H. An and M. Zhao, "Direct numerical simulation of flow around a surface-mounted finite square cylinder at low Reynolds numbers," Physics of Fluids, 29(4), 045101, 2017.

[18] J.A. Bourgeois, P. Sattari, R.J. Martinuzzi, "Alternating half-loop shedding in the turbulent wake of a finite surface-mounted square cylinder with a thin boundary layer," Physics of Fluids, 23 (095101), pp. 1-15, 2011.

[19] J. Brinkerho $\square$ and M.I. Yaras, "Numerical investigation of the generation and growth of coherent flow structures in a triggered turbulent spot," J. of Fluid Mechanics 759, pp. 257-294, 2014.

[20] M. Tobak and D.J. Peake, "Topology of three-dimensional separated flows," Ann. Rev. Fluid Mech., Vol. 14, pp. 61-85, 1982. 